\title{
Traffic noise: Annoyance assessment of real and virtual sounds based on close proximity measurements
}

\author{
${ }^{a}$ School of Engineering, Department of Civil Engineering, University of Minho, Portugal \\ ${ }^{\mathrm{b}}$ School of Psychology, Department of Basic Psychology, University of Minho, Portugal \\ ${ }^{\mathrm{c}}$ Centro de Território Ambiente e Construção, University of Minho, Portugal \\ ${ }^{\mathrm{d}}$ Centro Algoritmi, University of Minho, Portugal \\ ${ }^{\text {e }}$ Centro de Computação Gráfica, Portugal \\ ${ }^{\mathrm{f}}$ INESC TEC, Portugal
}

F. Soares ${ }^{\mathrm{a}, \mathrm{c}}$, E. Freitas ${ }^{\mathrm{a}, \mathrm{c}, *}$, C. Cunha $^{\mathrm{a}}$, C. Silva ${ }^{\mathrm{e}, \mathrm{f}}$, J. Lamas ${ }^{\mathrm{d}, \mathrm{e}}$, S. Mouta ${ }^{\mathrm{e}, \mathrm{f}}, \mathrm{JA}$. Santos ${ }^{\mathrm{b}, \mathrm{d}, \mathrm{e}}$

\section{A R T I C L E I N F O}

Article history:

\section{Keywords:}

Annoyance

Traffic noise

Psychoacoustic analysis

Virtual sounds

Close proximity

\begin{abstract}
A B S T R A C T
The negative impact of noise on human health is well established and a high percentage of environmental noise is related with traffic sources. In this study, we compared annoyance judgments of real and virtual traffic sounds. Virtual sounds were generated through an auralization software with input from close proximity tyre/road noise measurements and real sounds were recorded through a Head and Torso Simulator. Both groups had sounds generated at two speeds and from three urban pavement surfaces (asphalt concrete, concrete blocks and granite cubes). Under controlled laboratory conditions, participants rated the annoyance of each real and virtual stimulus.

It was found that virtual stimuli, based on close proximity tyre/road noise, can be used to assess traffic annoyance, in spite of systematic lower rates than those found for real stimuli. The effects of type of pavement and speed were the same for both conditions (real and virtualized stimulus). Opposed to granite cubes, asphalt concrete had lower annoyance rates for both test speeds and higher rate differences between real and virtual stimuli. Additionally, it was also found that annoyance is better described by Loudness than by LAmax. This evidence is stronger for the virtual stimuli condition than for the real stimuli one. Nevertheless, we should stress that it is possible to accurately predict real annoyance rates from virtual auralized sound samples through a simple transformation model.

The methodology developed is clearly efficient and significantly simplifies field procedures, allowing the reduction of experimental costs, a better control of variables and an increment on the accuracy of annoyance ratings.
\end{abstract}

(c) 2017 Elsevier Ltd. All rights reserved.

\section{Introduction}

Transportation is and will remain in the foreseeable future a major source of environmental noise. The negative impact of noise on human health is well established. In the western European countries the range of the burden disease due to environmental noise would be 1.0-1.6 million DALYs $1^{1}, 587,000$ years of which are related to traffic annoyance (WHO, 2011).

\footnotetext{
* Corresponding author at: Departamento de Engenharia Civil - Universidade do Minho, Campus de Azurém, 4800-058 Guimarães, Portugal. E-mail address: efreitas@civil.uminho.pt (E. Freitas).

1 The EBD is expressed as disability-adjusted life years (DALYs). DALYs are the sum of the potential years of life lost due to premature death and the equivalent years of "healthy" life lost by virtue of being in states of poor health or disability.
} 
In this context, several annoyance studies have been carried out over the last few years. The recent ones were more concerned with health-related quality of life as, for instance, studies on the annoyance caused by different noise sources, including road traffic noise exposure (Héritier et al., 2014) or on the noise annoyance of residential transportation related to physical activity and its change over time (Foraster et al., 2015).

Previous studies, more concerned with road-traffic noise generation, addressed the impact of pavement type on annoyance (Sandberg and Ejsmont, 2002 and Golebiewski et al., 2003). Other authors addressed annoyance taking into account traffic characteristics (Griefahn et al., 2008; Paunović et al., 2009), powered-two-wheelers and heavy vehicles (Gille and Marquis-Favre, 2015; Morel et al., 2016), but overlooking the influence of the type of road surface.

In the scope of road-traffic noise generation, the most recent studies analysed annoyance considering the interactions of several types of pavements with relevant traffic parameters such as speed, type of vehicle, and vehicle composition (Freitas et al., 2012). However, the main issue is that the experimental procedure to measure tyre-road noise is very complex and time-consuming. Moreover, the field recordings are subjected to contamination by other noise sources, thus limiting considerably the number and type of parameters which could be manipulated or even the feasibility of the studies. Therefore, to avoid these drawbacks, a more straightforward procedure that combines noise acquisition close to the tyre (close-proximity measurements) with auralization of these acquisitions in a virtual scenario, must be tested and validated. Moreover, by manipulating the sound measured in the source (the tyre), it will be possible to manipulate and analyse a wider set of traffic parameters and other road environment features.

Recently, the suitability of close proximity measurements was examined (Freitas et al., 2015). In this work, a relation between subjective annoyance ratings and traffic noise levels described by acoustic and psychoacoustic indicators (LAmax, LAeq and Loudness $)^{2}$ as a function of speed was established. However, the use of virtual sounds, built from close proximity records, to assess traffic annoyance is still not a fully explored area and results are insufficiently documented in the literature.

This paper intends to address two main questions: (1) ascertain if it is possible to use close proximity tyre road noise measurements to rate traffic annoyance, and (2) verify if virtual sounds generated from these noise measurements can be used to rate annoyance correctly.

Tyre-road noise Close Proximity (CPX) and Head and Torso Simulator (HATS) samples were recorded simultaneously from several combinations of pavement and speed. According to the Controlled Pass-By method (CPB) (Freitas et al., 2012), CPX samples were then edited and auralized to create realistic pass-by noise near the traffic lanes. This allowed generating the virtual stimuli. HATS recordings were used for the real stimuli.

Under controlled laboratory conditions, participants rated the annoyance of each real and virtual traffic noise in a withinsubject design. To better establish a relation between the subjective annoyance ratings of real and virtual traffic noise conditions, both traditional and psychoacoustic parameters were considered such as Lamax and Loudness. These parameters are not currently used with CPX measurements however in a previous study they showed a good correlation with annoyance ratings (Freitas et al., 2012). Furthermore, LAmax was preferred as acoustic parameter in the calibration procedure of CPX and CPB sounds, because it captures better the fast sound level variations in the CPB sounds.

\section{Materials and methods}

\subsection{Pavement surfaces}

The types of pavement surfaces selected for the study were: granite cubes, concrete blocks, and asphalt concrete with a maximum aggregate size of 16 [mm] (Fig. 1). Granite cubes and concrete blocks are frequently used in urban areas for aesthetical reasons and as a traffic calming measure, particularly in city centres. Asphalt concrete is used in a wide range of situations in both urban, rural, and highway roads. This kind of pavement is the most common type in western countries and thus it is often used as a reference surface.

\subsection{Recordings}

The tyre-road noise used for stimulus in the virtual stimuli condition was recorded with a Brüel \& Kjaer Pulse Analyzer type 3560-C and two microphones were assembled by the Close Proximity method (CPX) according to ISO/CD 11819-2. The sound recordings of the stimuli used in the real stimuli condition were obtained using a Brüel \& Kjaer Pulse Analyzer type 3560-C and a Brüel \& Kjaer Head and Torso Simulator (HATS) type 4128-C located at $7.5 \mathrm{~m}$ from the road centre and at a height of $1.7 \mathrm{~m}$ following the procedure adopted in previous studies (Freitas et al., 2012).

The same vehicle was used for both measurements at two different speeds - 30 and $50 \mathrm{~km} / \mathrm{h}$. The tyre used in the vehicle was the ContiEcoContact3 195/65-R15 with acceptable performance when compared to other recommended reference tyres (Morgan et al., 2009).

\footnotetext{
${ }^{2}$ Traffic noise and tyre/road noise are currently assessed by acoustic indicators in decibels ( $\mathrm{dB}$ ) as the A-weighted equivalent mean sound pressure level (LA, eq) and the A-weighted maximum sound pressure Level (LAmax). Psychoacoustic indicators are also used, such as Loudness. Loudness is the attribute of auditory sensation in terms of which sounds may be ordered on a scale extending from soft to loud. The 'loudness level' of a sound is defined as the sound pressure level of a $1 \mathrm{kHz}$ tone in a plane wave and frontal incident that is as loud as the sound; its unit is "phon". The sone scale is based on the observation that a 10 phon increase in a sound level is most often perceived as a doubling of loudness.
} 


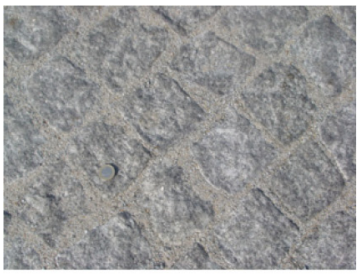

(a)

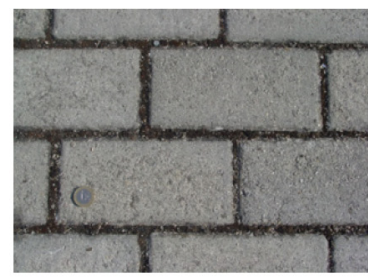

(b)

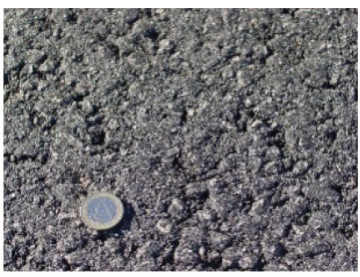

(c)

Fig. 1. Pavement surfaces: a) granite cubes (GC); b) concrete blocks (CB); c) asphalt concrete (AC).

In order to minimize any meteorological bias all recording sessions were performed with dry pavements, wind speed below $5 \mathrm{~m} / \mathrm{s}$, atmospheric temperature between $5^{\circ}$ and $30^{\circ} \mathrm{C}$.

\subsection{Pavement acoustic characterization}

The surface characteristics of the pavements under analysis are quite distinct. To better understand their effect on traffic noise see Sandberg and Ejsmont (2002). There are clear differences in physical characteristics between the dense asphalt and the concrete block pavements (Table 1 ), but these physical differences result in acoustic differences under $0.5 \mathrm{~dB}(\mathrm{~A}) \mathrm{for}$ the Lamax and 1.7 sone for the samples obtained by the Close Proximity method. Granite cubes generated approximately more $2 \mathrm{dBA}$ and more 5 sone than the other pavements. Loudness was more affected by speed than LAmax. This effect can be observed by the large difference between speed values of each type of surface.

\subsection{Method to construct virtual stimuli}

The virtual stimuli were constructed using as input noise sounds from tyre-road CPX measurements. The objective was to create a moving sound source similar to the CPB measurements with five seconds of length in which a vehicle passes-by the subject in a front-parallel plane. To achieve this, an auralization process was conducted using Head Related Transfer Functions (HRTFs). The original CPX sound was divided into small samples and, for each sample, a convolution was made between the sample and the correct HRTF (in time domain a HRIR - Head Related Impulse Response was used) depending on the sample position. For example, at $30 \mathrm{~km} / \mathrm{h}$, the vehicle at the beginning of the stimulus was located at approximately $28.83 \mathrm{~m}$ to the left of the participant and had an azimuth angle variation, throughout the presentation, between $-75^{\circ}$ and $75^{\circ}$. Thus, several HRTFs were selected between these angles according to the correct position of the sound source at the beginning of each small sample.

Since the final stimuli consist of moving sound sources, it was applied a Doppler effect to the final sounds. Depending on the speed and distance, a Doppler coefficient was calculated and a sound resample was made for each sample.

The geometrical attenuation applied in the virtualization of the moving sound source was based on a previous study (Anfosso-Lédée, 2004). The sound pressure level difference between the CPX measurement ( $\mathrm{L}_{\mathrm{CPX}}$ ) and the CPB measurement $\left(\mathrm{L}_{\mathrm{CPB}}\right)$ is defined as:

$$
\Delta L=L_{C P X}-L_{C P B}
$$

In this geometrical model it is assumed that a tyre source is approximated by a point monopole source $S_{t}$ which corresponds to the contact "point" between road surface and tyre. An equivalent point source $S_{\text {eq }}$ was used to define the vehicle as a unique sound source, as shown in Fig. 2.

The final equation of this geometrical model is given by:

$$
\Delta L=A t t_{C P X}-A t t_{c t}-20 \log _{10}\left(\frac{r_{C P X}}{r_{c t}}\right)-10 \log _{10}\left(1+\left(\frac{r_{c t}}{r_{f t}}\right)^{2} 10^{A^{A t t_{f}}-\text { Attct }^{10}}\right)-10 \log _{10}
$$

where $\mathrm{Att}_{\mathrm{CPX}}$ is the sound pressure attenuation between the CPB measurement position and the $\mathrm{S}_{\mathrm{eq}}$ position relative to the free field, Att $_{\mathrm{ct}}$ is the sound attenuation relative to free field between the closer tyres and the CPB measurement position, Att $\mathrm{ft}_{\mathrm{ft}}$ is the sound attenuation relative to free field between the further tyres and the CPB measurement position, $\mathrm{r}_{\mathrm{CPX}}$ is the distance between the CPX measurement position and the tyre source position, $\mathrm{r}_{\mathrm{ct}}$ is the distance between the closer tyres and the CPB measurement, and $\mathrm{r}_{\mathrm{ft}}$ is the distance between the further tyres and the CPB measurement.

The sound reflections on the car body were not considered in this stimuli synthesis. The noise from engine, exhaust, transmission and others vehicle sources were also disregarded. No filter representing the road surface attenuation was used due to the very low absorption of these surfaces, consequently the road was considered as a pure reflective road surface. In this case, the previous equation was simplified since for pure reflecting surfaces the $A_{\mathrm{ct}}=\mathrm{Att}_{\mathrm{ft}}=\mathrm{Att}_{\mathrm{CPX}}=6 \mathrm{~dB}$ for all frequencies. Therefore the final geometrical attenuation was a function dependent only on physical distances: 
Table 1

Left-right average of LAmax and Loudness obtained by CPX method.

\begin{tabular}{lllll}
\hline Type of surface & LAmax $[\mathrm{dB}(\mathrm{A})]$ & & Loudness [sone] \\
\cline { 2 - 3 } & $30[\mathrm{~km} / \mathrm{h}]$ & $50[\mathrm{~km} / \mathrm{h}]$ & $30[\mathrm{~km} / \mathrm{h}]$ & $50[\mathrm{~km} / \mathrm{h}]$ \\
\hline Granite cubes & 70.5 & 77.1 & 25.9 & 37.5 \\
Concrete blocks & 68.3 & 75.0 & 21.2 & 31.5 \\
Asphalt concrete & 68.5 & 74.6 & 20.0 & 28.7 \\
\hline
\end{tabular}

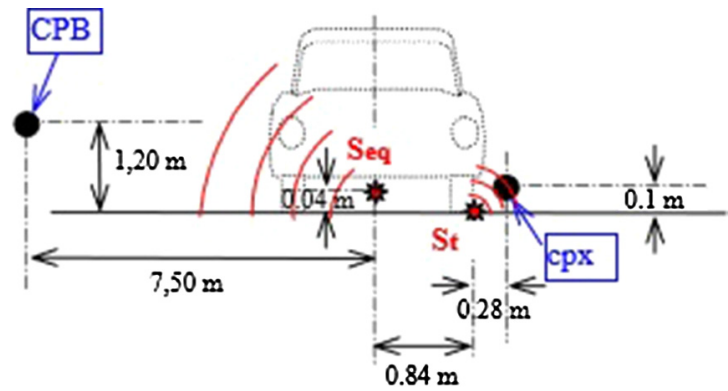

Fig. 2. Sources location (Anfosso-Lédée, 2004).
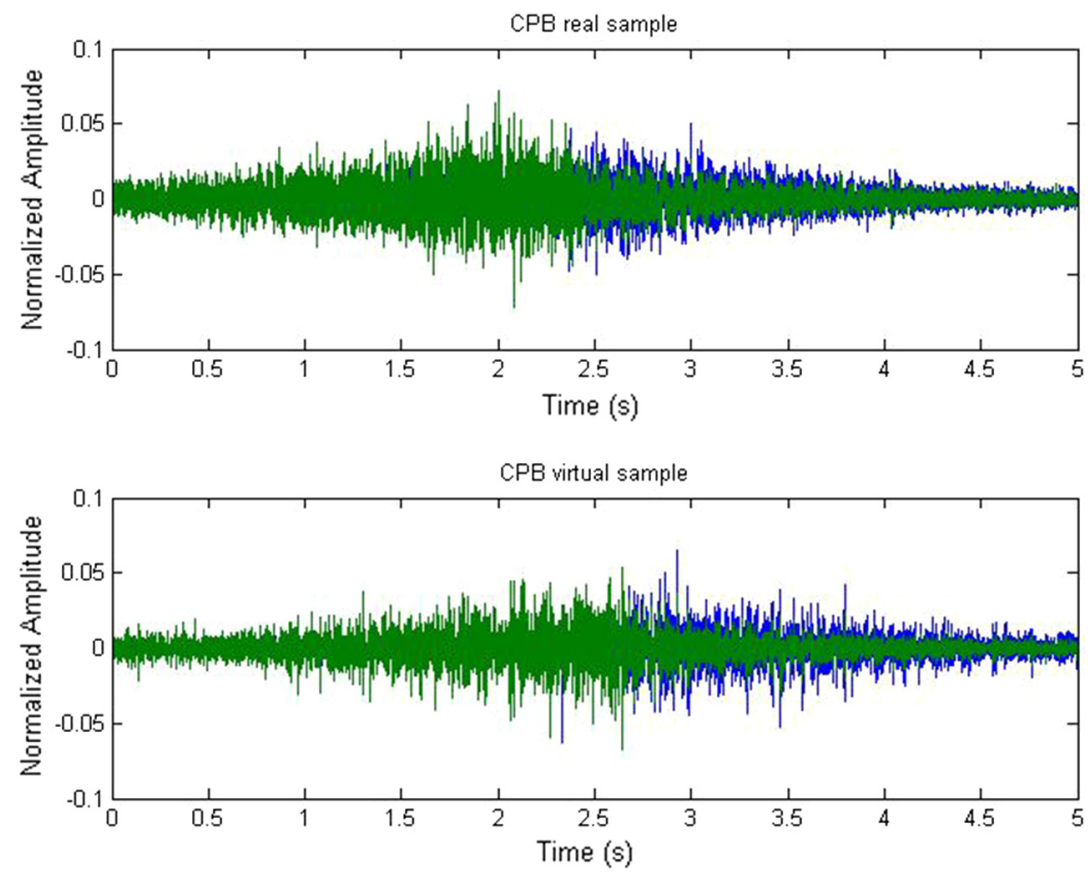

Fig. 3. Examples of stimuli characterized by intensity as a function of time. Green represents the left channel and blue represents the right channel. (For interpretation of the references to colour in this figure legend, the reader is referred to the web version of this article.)

$$
\Delta L=10 \log _{10}\left(\frac{r_{c t}^{2} r_{f t}^{2}}{2 r_{C P X}^{2}\left(r_{c t}^{2}+r_{f t}^{2}\right)}\right)
$$

In the final sound output a distance compensation was applied according to the distance between the vehicle and the position of the user, along the movement of the vehicle.

The two stimuli are depicted in Fig. 3: one original CPB recording with the HATS and one virtual stimulus generated with the above mentioned auralization algorithm. Both exemplificative signals represent the granite cubes surface at $50 \mathrm{~km} / \mathrm{h}$. 


\section{Experiment}

The experiment was designed to verify if close proximity tyre road noise measurements are suitable to rate traffic annoyance, and to analyse if virtual sounds generated from close proximity tyre road noise measurements (virtual stimuli) can be used to rate annoyance correctly.

\subsection{Participants}

Fifteen listeners participated in this experiment, eight female and seven male (average of $25 \pm 2.3$ years old). They were all voluntary participants, recruited via academic institutions in the geographical area where noise samples were recorded. All subjects had normal hearing as confirmed with an audiogram test on a Beltone 109 audiometer. Previous studies on noise annoyance assessment revealed a high inter-individual consistency of the results regardless of age, gender or cultural factors (Freitas et al., 2012). Therefore, the small sample size in this study, should not compromise the accuracy of the assessment.

\subsection{Stimuli}

Two types of stimuli, with a duration of five seconds per stimulus, were presented to the participants: a real stimuli database generated from the HATS recordings, and a virtual stimuli database generated from the manipulation of the CPX recordings.

Both stimuli were built with all possible combinations: speed $(30 \mathrm{~km} / \mathrm{h}$ and $50 \mathrm{~km} / \mathrm{h}$ ) and type of surface (granite cubes $(\mathrm{GC})$, concrete blocks $(\mathrm{CB})$ and asphalt concrete $(\mathrm{AC})$ ). The stimuli sounds (real and virtual) were also generated with two different movement directions: starting from the left to the right and vice versa.

The stimuli were presented to participants through a custom built $\mathrm{C}++$ application, running on a computer with an Intel 82801BA-ICH2 sound card and AKG K 271 MKII closed headphones. This system was calibrated to achieve sound pressure levels identical to those found in the original tyre-road measurements.

\subsection{Procedure}

The annoyance assessment of each participant was performed in a quiet room. Each sound condition (real or virtual) was presented channel reversed to avoid interaural biases and repeated ten times. Thus each participant listened to a total of 240 noise trials ( 2 conditions $\times 6$ stimuli $\times 2$ channel sequences $\times 10$ repetitions). Trials were blocked by type of stimuli in a pseudo-random order (method of the constant stimulus) to reduce anticipation and learning effects. Participants were requested to assess the annoyance of each noise trial with a 10-graded interval scale from 1 (less annoying) to 10 (very annoying). The interval between trials was variable and depended on the promptness of the participant: after the answer to a given trial (by pressing a number on a keyboard) the next noise sample was presented. Each block, with the 120 trials, lasted for about 14 min per participant.

\section{Results}

\subsection{Pavement, speed and type of stimuli}

All the participants' responses to the 240 stimuli were analysed to evaluate the effect of each variable on the annoyance assessment.

An analysis of variance through the application of a three-way ANOVA (3 factors: type of pavement, speed, and type of stimuli) was conducted to determine if there was an interaction effect between these three independent variables over annoyance ratings. Results are reported by F-value (result of the application of Fisher test), degree of freedom between groups $\left(\mathrm{df}_{1}\right)$, degree of freedom within groups $\left(\mathrm{df}_{2}\right)$ and significance level $(\mathrm{p})$ which indicates the risk (\%) of concluding that an effect exists when there is no actual effect. All of these parameters are simply presented as follows: $\left(F_{d f 1, d f 2}, p\right)$. Significant main effects over annoyance ratings were found by: type of pavement $\left(F_{2,3570}=2010.07, p<0.001\right)$; speed $\left(F_{1,3570}=719.92\right.$, $\mathrm{p}<0.01)$; stimulus type $\left(\mathrm{F}_{1,3570}=154.87, \mathrm{p}<0.001\right)$; with interaction effects between type of pavement and speed $\left(F_{2,3570}=26.75, p<0.001\right)$; type of pavement and type of stimulus $\left(F_{2,3570}=145.81, p<0.001\right)$; and type of surface, speed and type of stimulus $\left(F_{2,3570}=6.70, p<0.005\right)$. As expected, only the speed and type of stimulus interaction was not significant $\left(F_{1,3570}=0.119\right.$, n.s.). In addition, Tukey tests were carried out to verify if there were post-hoc differences between groups of each variable. These tests, with significance level $\left(\mathrm{p}_{\text {adj }}\right)$, revealed significant differences between all groups for pavement type, speed and type of stimuli ( $\left.p_{a d j}<0.001\right)$.

Additionally, in line with previous studies (Freitas et al., 2012), the analysis of the inter-participant data revealed a high consistency of annoyance rates as a function of the main variables (pavement, speed, stimuli type). Although the effect of participants for annoyance ratings differed significantly $\left(F_{14,3567}=59.6, \mathrm{p}<0.001\right)$, these differences were due to the absolute values for individual results, and consequently the magnitude of the ratings. The main trend regarding the effects of the main 
variables on annoyance rates were consistent between participants. For that reason, hereafter averaged data of the trials of all participants was used.

Fig. 4 shows box-plots for the average annoyance ratings for both stimuli types (real - R- and virtual - V - stimuli), both speeds (30 km/h - panel A; $50 \mathrm{~km} / \mathrm{h}$ - panel B) in all the pavement types. The bottom and top of each box represent respectively the 1 st and 3rd quartiles, the horizontal line inside each one shows the median for each stimuli type and the lines extending vertically from the boxes indicate the variability outside the upper and lower quartiles.

We found similar results for the real and virtual stimuli, with concrete blocks (CB) and granite cubes (GC). Conversely there is a noticeable difference of the mean annoyance for real and virtual stimuli with asphalt concrete (AC). While granite cubes (also called cobble stones) pavement induces the highest rate of annoyance; asphalt concrete induces the lowest one for both test speeds. Concrete blocks annoyance mean ratings are close to granite cubes ratings particularly at high speeds.

The significant speed-pavement interaction revealed in the three-way ANOVA, pointed to an increase in annoyance as a function of speed for all pavement types, as can be clearly seen in Fig. 4. Again, annoyance rates based on virtual stimuli show the lowest rates of annoyance confirming the speed-type of stimulus interactions. Pavement- stimulus type interactions are particularly visible in Fig. 4 for asphalt concrete with virtual stimuli mean ratings considerably lower than real ratings.

The aforementioned results indicate that for both speeds there is a similar trend between the annoyance rate and the type of pavement. This trend is visible either for real or virtual stimuli (see Fig. 4). Thus, it is expectable to find a consistent ratio between annoyance rates of both stimuli for the two test speeds and three surfaces, as is clearly shown in Fig. 5 .

Therefore, considering the testing conditions, real annoyance ratings (Ran) can be predicted $\left(R^{2}=0.915\right)$ as a function of the annoyance ratings obtained through virtual stimuli (Van) by the equation:

$$
\text { Ran }=2.606+0.624 \text { Van }
$$

\subsection{Annoyance and acoustic indicators}

To better understand, predict, and compare how different pavements influence annoyance ratings, several acoustic measurements were evaluated, namely the widely used Lamax and Loudness D, being the latter the best tyre-road noise predictor (Freitas et al., 2012). In Figs. 6 and 7, LAmax and Loudness analyses are presented.

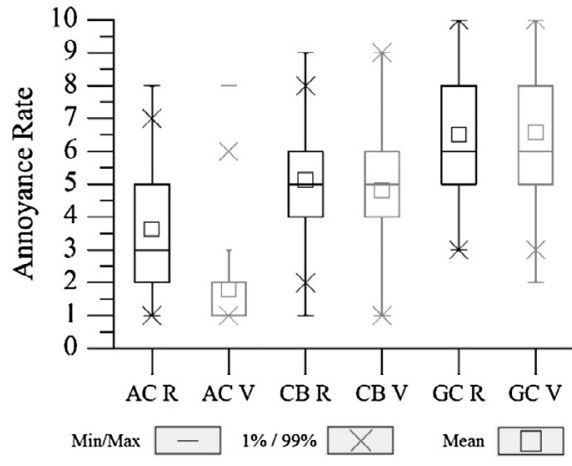

(a)

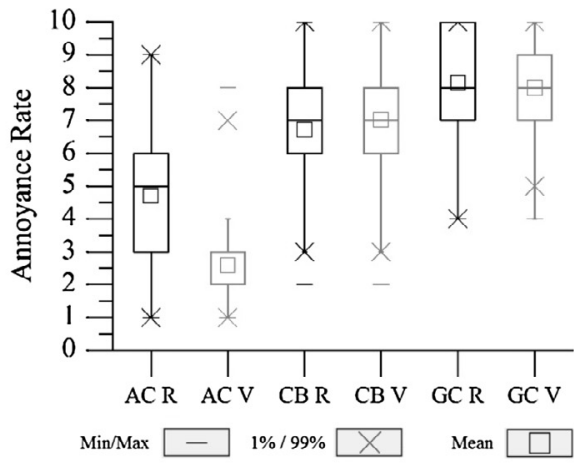

(b)

Fig. 4. Annoyance ratings box-plot for real (R) and virtual (V) stimuli per pavement type (AC, CB and GC) at: (a) $30 \mathrm{~km} / \mathrm{h}$ and (b) $50 \mathrm{~km} / \mathrm{h}$.

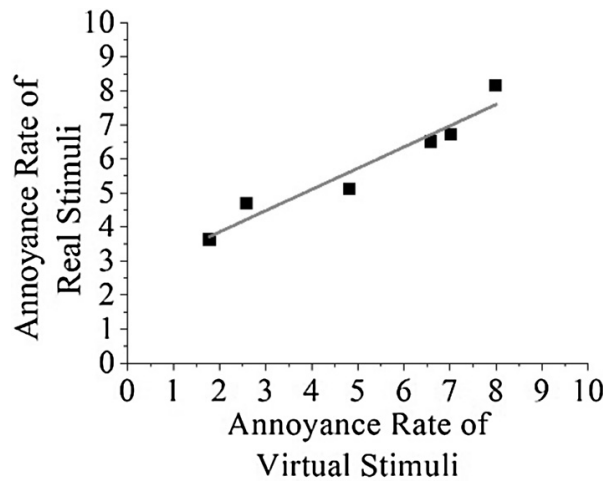

Fig. 5. Linear relation between annoyance ratings of real stimuli and virtual stimuli (averaged data $\mathrm{n}=300$ ). 


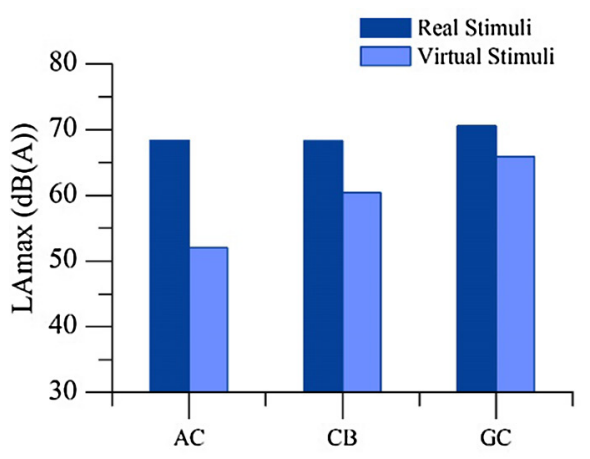

(a)

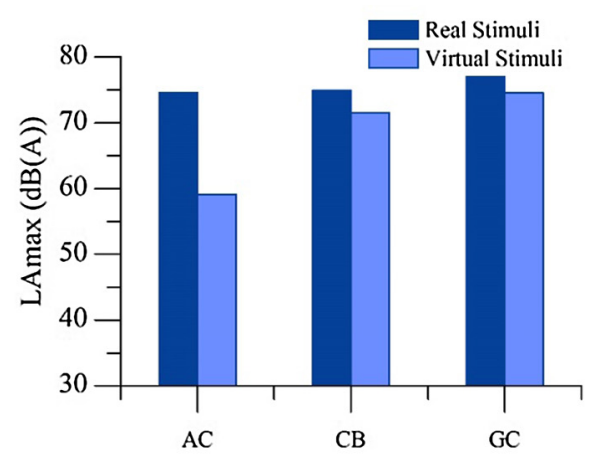

(b)

Fig. 6. LAmax results per type of stimuli and pavement at: (a) $30 \mathrm{~km} / \mathrm{h}$ and (b) $50 \mathrm{~km} / \mathrm{h}$.

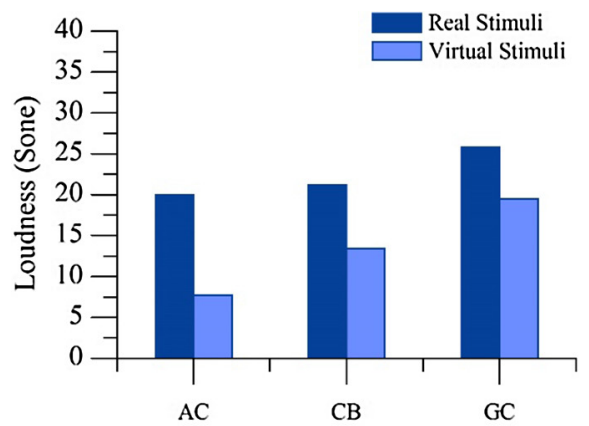

(a)

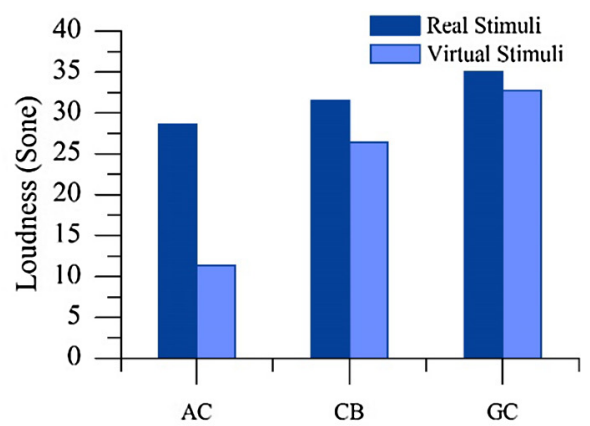

(b)

Fig. 7. Loudness D results per type of stimuli and at: (a) $30 \mathrm{~km} / \mathrm{h}$ and (b) $50 \mathrm{~km} / \mathrm{h}$.

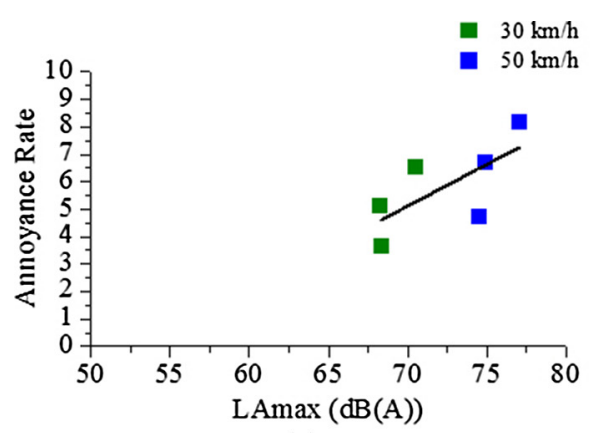

(a)

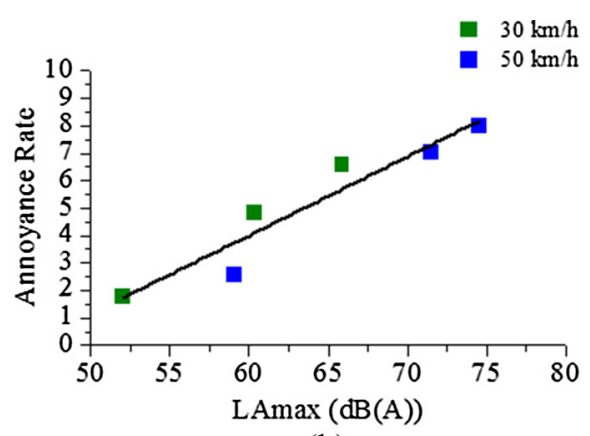

(b)

Fig. 8. Mean annoyance ratings versus LAmax (averaged data $n=300$ ): (a) real stimuli and (b) virtual stimuli.

Once more, the difference in the sensitivity of the acoustic indicators for both real and virtual stimuli is clear. The acoustic indicators calculated from virtual stimuli are more sensitive to the effect of type of pavement, particularly of the asphalt concrete. Nevertheless, Loudness reflects differences among each type of surface better than LAmax, and again more perceptible for the asphalt concrete. As expected, increasing speed leads to higher values of both acoustic indicators.

In Fig. 8, LAmax and annoyance rates analyses are presented. The averaged annoyance rates for all pavements are well predicted by the LAmax only for virtual stimuli $\left(r^{2}=0.89\right.$, slope 0.29$)$. For real stimuli, the LAmax explains only 35\% of the annoyance rates $\left(r^{2}=0.35\right.$, slope 0.30$)$. Conversely, the Loudness D explains the annoyance rates (Fig. 9) for real stimuli $\left(r^{2}=0.64\right.$, slope $\left.=0.21\right)$ and for virtual stimuli $\left(r^{2}=0.85\right.$, slope $\left.=0.25\right)$. The annoyance prediction for the later can be further improved through a linear fit with the logarithm of loudness $\left(r^{2}=0.93\right.$, slope $\left.=10.35\right)$. 


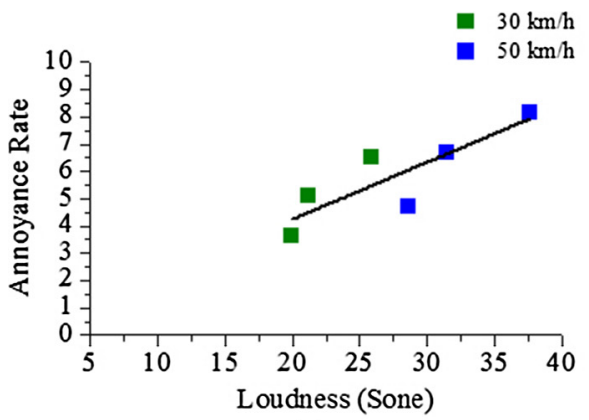

(a)

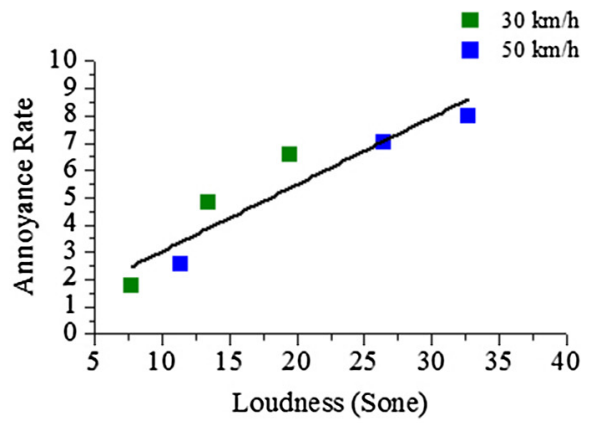

(b)

Fig. 9. Mean annoyance ratings as function of Loudness $D$ (averaged data $n=300$ ): (a) real stimuli, (b) virtual stimuli.

\section{Discussion and conclusions}

From our experimental results it is clear that virtual stimuli generated from close proximity tyre road noise measurements can be used to rate traffic annoyance.

The advantages of using virtual instead of real stimuli are several, including a better control and a faster implementation of experimental factors associated with lower costs. Real-virtual data differences can be explained in part by the simplified sound propagation algorithm used. This model can be improved, for example, by analyzing non-reflective surfaces. Nevertheless, we should stress that our approach allows to strictly assess the tyre/road noise and related annoyance, with no interferences. In a matter of fact, virtual stimuli are composed only of tyre-road noise and are free of interferences from engine, mechanical and environmental noise. In the range of testing speeds $(30 \mathrm{~km} / \mathrm{h}$ and $50 \mathrm{~km} / \mathrm{h}$ ) vehicle noises may dominate for asphalt concrete due to its surface characteristic, which is not the case of granite cubes and concrete blocks because they generate higher levels of tyre-road noise. Consequently, the following arguments may be drawn: (1) by ignoring vehicle mechanical noises it is possible to generate virtual stimuli based on tyre-road noise only, which can be used to infer about annoyance rates induced by quieter electrical and hybrid vehicles, for example; (2) real stimuli seem to contaminate participants' judgment on tyre-road noise coming from low noise surfaces.

Additionally, real-virtual differences are expressed mainly by higher annoyance rates for real stimuli, which can be predicted by a simple linear model. The model predicts real ratings as a function of virtual stimuli annoyance rates and accounts both for the effect of speed and type of pavement.

Loudness predicts annoyance rates and reflects differences among each type of surface better than the widely used acoustic indicator LAmax. Once more, the quality of the prediction is higher for virtual stimuli. These findings strengthen the fact that road traffic annoyance studies and requirements will benefit from the adoption of psychoacoustic indicators.

The outputs of this work have obvious practical implications for further studies on traffic noise and factors affecting annoyance. The methodology is efficient and significantly simplifies field procedures. Specifically, this approach reduces experimental costs, provides a better control of variables, and can increase the accuracy of annoyance ratings.

\section{Acknowledgements}

This work was financed by FEDER grants through the Operational Competitiveness Program - COMPETE and ON.2 - Novo Norte (Programa Operacional Regional do Norte 2007/2013) integrated in the structural funds QREN and the project PEst-OE/ ECI/UI4047/2014 supported by Portuguese Foundation for Science and Technology.

\section{References}

Anfosso-Lédée, F., 2004. Modeling the local propagation effects of tire-road noise: propagation filter between CPX and CPB measurements. Inter.noise, Prague, 22-25 August.

Foraster, M., Eze, I., Vienneau, D., Brink, M., Cajochen, C., Héritier, H., Wunderli, J., Röösli, M., Probst-Hensch, N., 2015. Annoyance to Transportation Noise and Risk of Physical Inactivity. EuroNoise, Maastricht, 31 May-3 June.

Freitas, E., Mendonça, C., Santos, J.A., Murteira, C., Ferreira, J.P., 2012. Traffic noise abatement: how different pavements, vehicle speeds and traffic densities affect annoyance levels. Transport. Res. Part D: Transport Environ. 17 (4), 321-326.

Freitas, E., Cunha, C., Lamas, J., Mouta, S., Santos, J.A., 2015. A Psychoacoustic Based Approach to Pavement Classification. Euronoise, 2015,31 May - 3 June. Maastricht, Netherlands.

Gille, L., Marquis-Favre, C., 2015. Does the order of different successive vehicle pass-bys have an influence on the annoyance due to an urban road traffic noise?. EuroNoise, Maastricht, 31 May-3 June.

Golebiewski, R., Makarewicz, R., Nowak, M., Preis, A., 2003. Traffic noise reduction due to the porous road surface. Appl. Acoust. 64, 481-494.

Griefahn, B., Gjestland, T., Preis, A., 2008. Annoyance of residents living in urban areas. Report to partners Deliverable A.D6: month 36 SILENCE Integrated Project - Contract N. 516288: European Commission.

Héritier, H., Vienneau, D., Frei, P., Eze, I., Brink, M., Probst-Hensch, N., Röösli, M., 2014. The association between road traffic noise exposure, annoyance and health-related quality of life (HRQOL). Int. J. Environ. Res. Public Health 11, 12652-12667. 
Morgan, P., Sandberg, U., Blokland, G., 2009: The Selection of New Reference Test Tyres for use with the CPX Method, to be Specified in ISO/TS 11819-3. Proc. INTER-NOISE 2009, Ottawa.

Morel, J., Marquis-Favre, C., Gille, L.A., 2016. Noise annoyance assessment of various urban road vehicle pass-by noises in isolation and combined with industrial noise: a laboratory study. Appl. Acoust. 101, 47-57. http://dx.doi.org/10.1016/j.apacoust.2015.07.017.

Paunović, K., Jakovljević, B., Belojević, G., 2009. Predictors of noise annoyance in noisy and quiet urban streets. Sci. Total Environ. 407, $3707-3711$.

Sandberg, U., Ejsmont, J., 2002. Tyre-Road Noise Reference Book. Informex SE-59040 Kisa: Sweden.

WHO, 2011. Burden of Disease From Environmental Noise. World Health Organization, Regional Office for Europe. 